\title{
Risk of COPD with obstruction in active smokers with normal spirometry and reduced diffusion capacity
}

\author{
Ben-Gary Harvey ${ }^{1,2,4}$, Yael Strulovici-Barel ${ }^{1,4}$, Robert J. Kaner ${ }^{1,2}$, \\ Abraham Sanders ${ }^{2}$, Thomas L. Vincent ${ }^{1}$, Jason G. Mezey ${ }^{1,3}$ and Ronald G. Crystal ${ }^{1,2}$ \\ Affiliations: ${ }^{1}$ Dept of Genetic Medicine, Weill Cornell Medical College, New York, NY, USA. ${ }^{2}$ Division of \\ Pulmonary and Critical Care Medicine, Dept of Medicine, Weill Cornell Medical College, New York, NY, USA. \\ ${ }^{3}$ Dept of Biological Statistics and Computational Biology, Cornell University, Ithaca, NY, USA. ${ }^{4}$ These authors \\ contributed equally to this study.
}

Correspondence: Ronald G. Crystal, Department of Genetic Medicine, Weill Cornell Medical College, 1300 York Avenue, Box 164, New York, NY 10065, USA. E-mail: geneticmedicinelamed.cornell.edu

ABSTRACT Smokers are assessed for chronic obstructive pulmonary disease (COPD) using spirometry, with COPD defined by the Global Initiative for Chronic Obstructive Lung Disease (GOLD) as airflow limitation that is not fully reversible with bronchodilators. There is a subset of smokers with normal spirometry (by GOLD criteria), who have a low diffusing capacity of the lung for carbon monoxide (DLCO), a parameter linked to emphysema and small airway disease. The natural history of these "normal spirometry/low DLCO” smokers is unknown.

From a cohort of 1570 smokers in the New York City metropolitian area, all of whom had normal spirometry, two groups were randomly selected for lung function follow-up: smokers with normal spirometry/normal DLCO $(\mathrm{n}=59)$ and smokers with normal spirometry/low DLCO $(\mathrm{n}=46)$. All had normal history, physical examination, complete blood count, urinalysis, HIV status, $\alpha_{1}$-antitrypsin level, chest radiography, forced expiratory volume in $1 \mathrm{~s}$ (FEV1), forced vital capacity (FVC), FEV1/FVC ratio and total lung capacity. Throughout the study, all continued to be active smokers.

In the normal spirometry/normal DLCO group assessed over $45 \pm 20$ months, 3\% developed GOLDdefined COPD. In contrast, in the normal spirometry/low DLCO group, followed over $41 \pm 31$ months, $22 \%$ developed GOLD-defined COPD.

Despite appearing "normal" according to GOLD, smokers with normal spirometry but low DLCO are at significant risk of developing COPD with obstruction to airflow.

@ERSpublications

Smokers with normal spirometry but low DLCO have a higher risk of COPD than smokers with normal spirometry and DLCO http://ow.ly/RWzxB

For editorial comments see Eur Respir J 2015; 46: 1535-1537 [DOI: 10.1183/09031936.01436-2015]

This article has supplementary material available from erj.ersjournals.com

Received: Dec 302014 | Accepted after revision: July 302015 | First published online: Nov 052015

Support statement: The studies were supported, in part, by US National Institutes of Health grants P50 HL084936 and UL1-RR024996. B-G. Harvey and R.J. Kaner were supported, in part, by the J.P. Smith Clinical Scholar Program. Funding information for this article has been deposited with FundRef.

Conflict of Interest: None declared.

Copyright OERS 2015 


\section{Introduction}

Chronic obstructive pulmonary disease (COPD), the third leading cause of mortality in the USA and Europe, is caused primarily by cigarette smoking [1-3]. The Global Initiative for Chronic Obstructive Lung Disease (GOLD) defines COPD as a chronic disease state characterised by airflow limitation that is not fully reversible with bronchodilators $[1,2]$. The GOLD criteria classify COPD into four stages based on post-bronchodilator forced expiratory volume in $1 \mathrm{~s}$ (FEV1) and forced vital capacity (FVC) [2]. With these criteria, if smokers have normal post-bronchodilator spirometry, they are considered to have normal lung function. While the evaluating physician will counsel the patient to stop smoking, the normal post-bronchodilator spirometry reassures both the patient and the physician that the patient does not have COPD and is at no greater risk of COPD than other smokers with normal post-bronchodilator spirometry.

Although the GOLD criteria are widely used [1, 4-6], it has been recognised that some smokers with normal spirometry have low diffusing capacity of the lung for carbon monoxide (DLCO), a parameter associated with alveolar destruction and possibly small airway disease, both of which are components of COPD [7-10]. DLCO measurement is not part of the GOLD criteria and is not used as a routine screening tool because of the lack of portability, the cost of the equipment, the expertise needed to carry out the measurement and the time involved $[1,11]$.

In the context that COPD is associated with both airway and alveolar disease [8], we asked: are smokers with normal post-bronchodilator spirometry but low DLCO at greater risk of developing COPD than smokers with normal post-bronchodilator spirometry and normal DLCO? To answer this question, we evaluated a group of cigarette smokers who answered advertisements in the New York metropolitan region for assessment of lung health. After clinical assessment, we characterised two groups: "normal spirometry/ low DLCO", smokers with normal post-bronchodilator spirometry and total lung capacity (TLC) but low DLCO; and control "normal spirometry/normal DLCO", smokers with normal post-bronchodilator spirometry, normal TLC and normal DLCO. A randomly chosen subset of these groups were asked to return for repeated lung function over time. Strikingly, with an average follow-up of $<4$ years, compared to smokers with normal spirometry/normal DLCO, a significant number of smokers in the normal spirometry/low DLCO group developed GOLD criteria-defined COPD, i.e. smokers who have normal post-bronchodilator spirometry but low DLCO are at a higher risk of developing COPD with obstruction to airflow compared to smokers with normal post-bronchodilator and normal DLCO.

\section{Methods}

Recruitment, screening and pulmonary function tests

Smokers were recruited from the New York metropolitan area via advertisements in newspapers and on websites under a protocol approved by the Weill Cornell Medical College and New York/Presbyterian Hospital Institutional Review Board. Healthy nonsmokers were also recruited to calculate the 95\% normal range for pulmonary function tests (PFTs) [12]. All individuals gave their informed written consent prior to any clinical evaluations or procedures. The study population was randomly chosen, using screening assessment and inclusion and exclusion criteria as detailed in the online supplementary material. PFTs were performed according to American Thoracic Society (ATS)/European Respiratory Society (ERS) standards [11, 13], and PFT machine calibrations were performed at the recommended intervals as described in the ATS/ERS guidelines [11] (online supplementary material).

\section{Study groups and assessment}

A total of 2302 active smokers were assessed. Based on the inclusion/exclusion criteria, a subset of 1570 active smokers were determined to be eligible. Of these, 1173 were phenotyped as normal spirometry/ normal DLCO and 397 as normal spirometry/low DLCO based on their DLCO prediction values (online supplementary material). A subset of these individuals were randomly contacted and asked to return for additional PFT assessments. The groups assessed over time included 59 smokers with normal spirometry/ normal DLCO and 46 smokers with normal spirometry/low DLCO (online supplementary table I).

\section{Statistical analysis}

Statistical analysis was performed as detailed in the online supplementary material.

\section{Role of the funding source}

The funding sources of the study had no role in study design, data collection, data analysis, data interpretation, or writing of the report or the decision to submit this report for publication. 


\section{Results}

Study population

Both the normal spirometry/normal DLCO and the normal spirometry/low DLCO groups had a preponderance of males and individuals of African-American descent, but had a similar distribution of sex, age and ethnicity (table 1). The two groups were assessed over a similar time period (online supplementary figure 1) and the age at the last assessment was similar ( $49 \pm 8$ versus $50 \pm 9$ years, respectively; $\mathrm{p}>0.9$ ); there were no differences in the smoking history, cough or sputum scores, Modified Medical Research Council

\section{TABLE 1 Demographics of study groups at baseline}

\begin{tabular}{|c|c|c|c|}
\hline \multirow[t]{2}{*}{ Parameter } & \multicolumn{2}{|c|}{ Smokers with normal spirometry } & \multirow[t]{2}{*}{ p-value } \\
\hline & Normal DLco & Low DLco & \\
\hline Individuals & 59 & 46 & \\
\hline Males/females & $43 / 16$ & $31 / 15$ & $>0.6$ \\
\hline Age years & $45 \pm 8$ & $46 \pm 8$ & $>0.5$ \\
\hline Ethnicity AA/E/H & $41 / 10 / 8$ & $37 / 5 / 4$ & $>0.6$ \\
\hline BMI $\mathrm{kg} \cdot \mathrm{m}^{-2}$ & $28 \pm 5$ & $25 \pm 5$ & $<0.002$ \\
\hline \multicolumn{4}{|l|}{ Smoking history" } \\
\hline Pack-years & $24 \pm 13$ & $30 \pm 15$ & $>0.05$ \\
\hline Packs per day & $1.0 \pm 0.5$ & $1.1 \pm 0.6$ & $>0.5$ \\
\hline Age of smoking initiation years & $17 \pm 5$ & $17 \pm 4$ & $>0.9$ \\
\hline Urine nicotine $\mathrm{ng} \cdot \mathrm{mL}^{-1}$ & $1102 \pm 1290$ & $951 \pm 1285$ & $>0.6$ \\
\hline Urine cotinine $\mathrm{ng} \cdot \mathrm{mL}^{-1}$ & $1276 \pm 927$ & $1298 \pm 894$ & $>0.9$ \\
\hline Cough score ${ }^{\pi}$ & $1.2 \pm 1.3$ & $1.7 \pm 1.5$ & $>0.06$ \\
\hline Sputum score ${ }^{\pi}$ & $1.1 \pm 1.3$ & $1.3 \pm 1.3$ & $>0.3$ \\
\hline MMRC score & $0.4 \pm 0.6$ & $0.5 \pm 0.6$ & $>0.2$ \\
\hline Emphysema ${ }^{+} \%$ & $2.0 \pm 0.02$ & $2.2 \pm 0.04$ & $>0.8$ \\
\hline \multicolumn{4}{|l|}{ Serology $y^{\S}$} \\
\hline$\alpha_{1}$-antitrypsin $\mathrm{mg} \cdot \mathrm{dL}^{-1}$ & $152 \pm 24$ & $145 \pm 21$ & $>0.1$ \\
\hline $\mathrm{ESR} \mathrm{mm} \cdot \mathrm{h}^{-1}$ & $13 \pm 11$ & $12 \pm 10$ & $>0.7$ \\
\hline $\lg E I U \cdot m^{-1}$ & $129 \pm 208$ & $169 \pm 259$ & $>0.4$ \\
\hline $\mathrm{CRP} \mathrm{mg} \cdot \mathrm{dL}^{-1}$ & $0.2 \pm 0.2$ & $0.3 \pm 0.2$ & $<0.005$ \\
\hline Hepatitis C negative/positive ${ }^{f}$ & $46 / 9$ & $39 / 6$ & $>0.8$ \\
\hline \multicolumn{4}{|l|}{ Lung function $\# \#$} \\
\hline VC \% predicted & $114 \pm 14$ & $108 \pm 14$ & $<0.05$ \\
\hline FVC \% predicted & $111 \pm 14$ & $104 \pm 14$ & $>0.1$ \\
\hline $\mathrm{FEV}_{1} \%$ predicted & $111 \pm 15$ & $104 \pm 14$ & $<0.03$ \\
\hline FEV $1 / F V C \%$ observed & $81 \pm 4$ & $79 \pm 5$ & $<0.03$ \\
\hline TLC $\%$ predicted & $99 \pm 13$ & $94 \pm 14$ & $<0.03$ \\
\hline RV \% predicted & $90 \pm 25$ & $89 \pm 37$ & $>0.8$ \\
\hline RV/TLC \% predicted & $28 \pm 7$ & $31 \pm 11$ & $>0.1$ \\
\hline DLco \% predicted & $93 \pm 10$ & $68 \pm 9$ & $<10^{-4}$ \\
\hline$D \mathrm{LCO} / V_{\mathrm{A}} \mathrm{mL} \cdot \mathrm{mHg}^{-1} \cdot \mathrm{min}^{-1} \cdot \mathrm{L}^{-1}$ & $4.4 \pm 0.6$ & $3.6 \pm 0.7$ & $<10^{-6}$ \\
\hline \multicolumn{4}{|c|}{ Assessment over time mean \pm so (range) } \\
\hline Duration of follow-up months & $46 \pm 21(5-113)$ & $41 \pm 31(5-146)$ & $>0.4$ \\
\hline Number of PFTs & $2 \pm 1(2-6)$ & $3 \pm 2(2-8)$ & $<10^{-3}$ \\
\hline Interval between PFTs months & $33 \pm 18(5-73)$ & $18 \pm 20(1-127)$ & $<10^{-6}$ \\
\hline
\end{tabular}

Data are presented as $\mathrm{n}$ or mean $\pm \mathrm{SD}$, unless otherwise stated. A total of 105 active smokers was enrolled in the study, including 46 individuals with normal history, and physical and general laboratory tests, normal posterior-anterior and lateral chest radiography, and normal spirometry and lung volumes, but low diffusing capacity of the lung for carbon monoxide ( $D\llcorner C 0)$, and 59 with normal spirometry, lung volumes and DLco. All were followed over time with full lung function studies. AA: African-American; E: European; $\mathrm{H}$ : Hispanic; BMI: body mass index; MMRC: Modified Medical Research Council dyspnoea scale [14]; ESR: erythrocyte sedimentation rate; CRP: C-reactive protein; VC: vital capacity; FVC: forced vital capacity; FEV1: forced expiratory volume in $1 \mathrm{~s}$; TLC: total lung capacity; RV: residual volume; VA: alveolar volume; PFT: pulmonary function test. \#: current smoking was verified at baseline by urine nicotine and its derivative cotinine; at subsequent visits for lung function testing, active smoking status was verified by questionnaire. ": cough and sputum scores were each evaluated on a scale of $0-4$, where 0 represented "not at all", 1 "only with chest infections", 2 "a few days a month", 3 "several days a week" and 4 "most days a week" [15]. ${ }^{+}$: chest high-resolution computed tomography \% emphysema at -950 Hounsfield units. ${ }^{\$}$ : all individuals tested negative for HIV and had normal levels of $\alpha_{1}$-antitrypsin. ${ }^{f}$ : data available for 55 out of 59 smokers

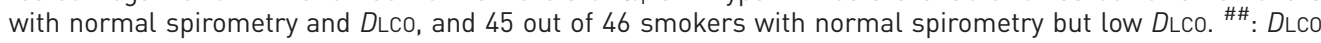
corrected for haemoglobin and carboxyhaemoglobin [11]. 

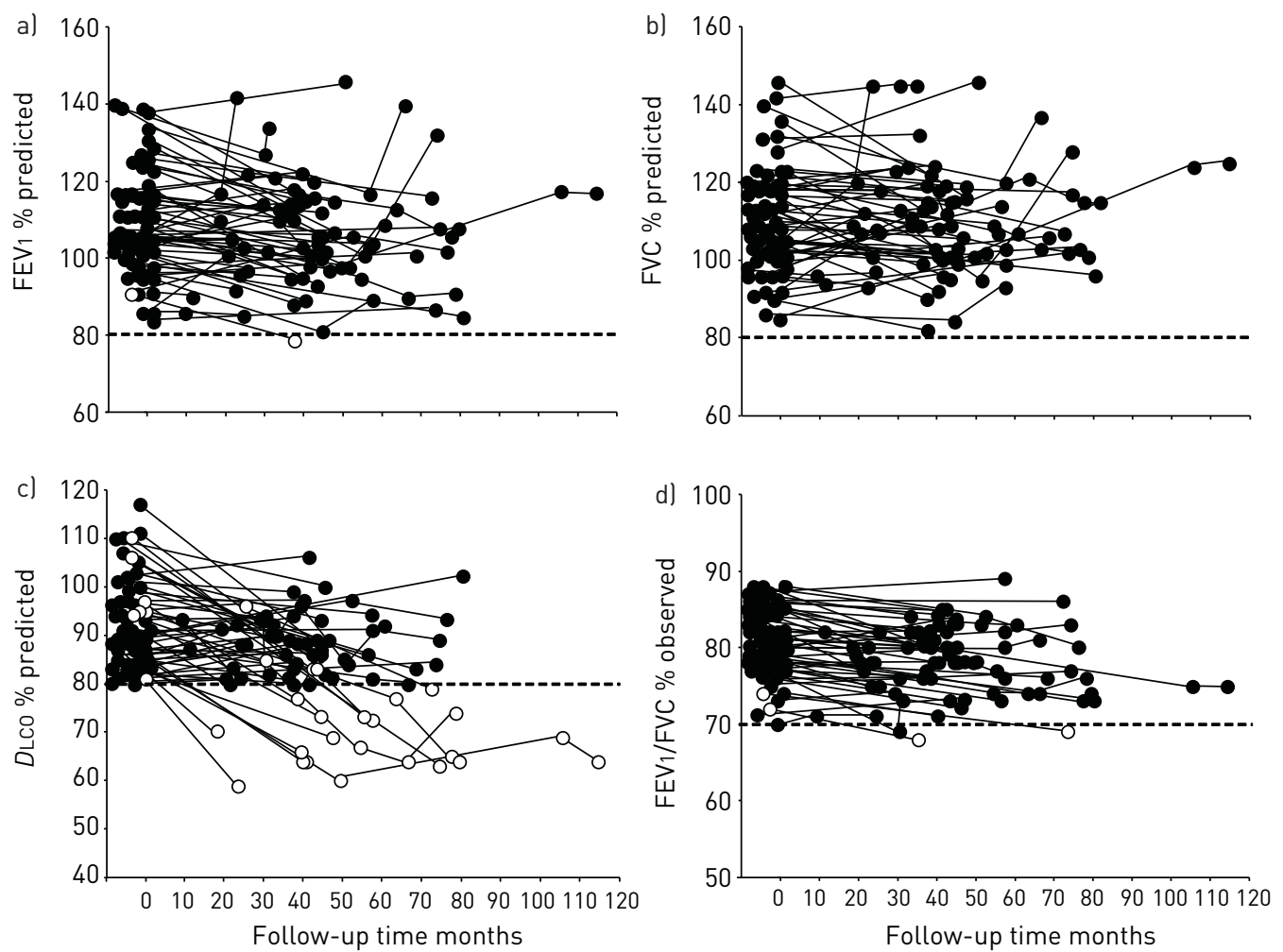

FIGURE 1 Lung function assessment over time of 59 active smokers with baseline normal history, physical examination and laboratory tests, and with normal spirometry, lung volumes, and normal diffusing capacity of the lung for carbon monoside ( $D\llcorner c 0)$. The abscissa shows time in months. Each symbol represents an individual, with lines connecting the follow-up data over time for the same individual. The dashed lines represent the lower limit of normal. Open circles indicate individuals that initially had normal values at baseline but became abnormal over time. Filled circles indicate individuals that had normal values at baseline and remained normal over time. a) Forced expiratory volume in $1 \mathrm{~s}\left(\mathrm{FEV}_{1}\right)$; b) forced vital capacity (FVC); c) DLCo; d) FEV1/FVC \% observed.

dyspnoea (MMRC) scale, or urine nicotine and cotinine levels between the two groups ( $\mathrm{p}>0.05$ for all comparisons). Percentage emphysema as assessesd by quantitative high-resolution computed tomography (HRCT) was not significantly different between the groups $(\mathrm{p}>0.8)$ (online supplementary figure 2). Except for slightly higher C-reactive protein levels in the normal spirometry/low DLCO group, other serology (erythrocyte sedimentation rate, IgE level and hepatitis C positivity/negativity) were not significantly different between the groups ( $p>0.1$ for all comparisons). Body mass index was lower in the normal spirometry/low DLCO group $(\mathrm{p}<0.002)$. Comparison of the lung function assessment between the two groups revealed, by definition, a difference in DLCO and DLCO/alveolar volume $\left(\mathrm{p}<10^{-4}\right.$ for both comparisons). Of the other PFT parameters evaluated, all were within normal range, with the normal spirometry/low DLCO group having a normal but lower vital capacity, FEV1, FEV1/FVC and TLC ( $p<0.03$ for all comparisons). When the groups were divided into African-American, European and Hispanic descendants, there was no significant difference attributed to ethnicity in any of the above parameters within the groups or between the groups ( $p>0.05$ and all comparisons).

\section{Lung function over time}

In the normal spirometry/normal DLCO group, the FEV1 \% predicted remained normal in 58 out of 59 individuals and the FVC \% predicted remained normal in all 59 individuals throughout the follow-up period (figure 1a and b). The DLCO in this group remained normal in 44 (75\%) out of 59 individuals but, interestingly, decreased to the normal spirometry/low DLCO category (DLCO $<80 \%$ predicted) in $15(25 \%)$ out of 59 individuals, suggesting that a significant number of active smokers with normal spirometry/ normal DLCO will progress to have low DLCO over an average of $<4$ years (figure 1c). Only two (3\%) out of the 59 active smokers in the normal spirometry/normal DLCO group developed COPD stage I as defined by the GOLD criteria [3] (FEV1/FVC $<0.7$ and $\mathrm{FEV}_{1} \geqslant 80 \%$ predicted, post-bronchodilators), one individuals at month 34 and the second at month 72 from baseline (figure 1d).

In the normal spirometry/low DLCO group, the FEV1 \% predicted remained normal in 44 out of 46 individuals and the FVC \% predicted remained normal in all 46 individuals (figure $2 \mathrm{a}$ and b). The DLCO 

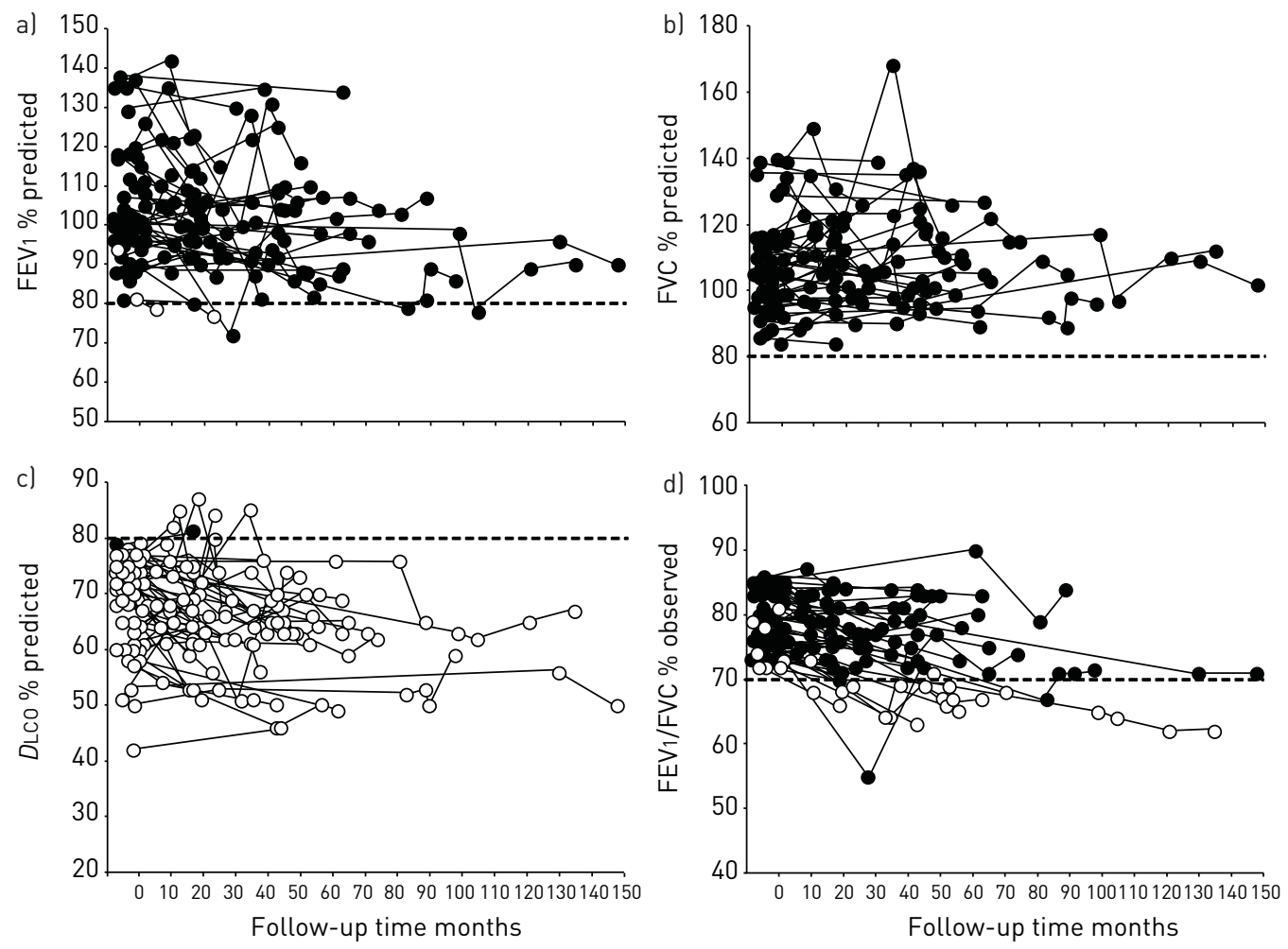

FIGURE 2 Lung function assessment over time in 46 active smokers with normal history, physical examination and laboratory tests, and with normal spirometry, lung volumes, but low diffusing capacity of the lung for carbon monoxide $(D\llcorner C 0)$. The abscissa shows time in months. Each symbol represents an individual, with lines connecting the follow-up data over time for the same individual. The dashed lines represent the lower limit of normal. Open circles indicate individuals that initially had normal values but became abnormal over time. Filled circles indicate individuals that had normal values at baseline and remained normal over time. a) Forced expiratory volume in $1 \mathrm{~s}\left(F_{E V}\right)$; b) forced vital capacity (FVC); c) DLCo; d) FEV $1 / F V C \%$ observed.

in this group remained low ( $<80 \%$ predicted) in 45 out of of 46 individuals (figure $2 \mathrm{c}$ ). In contrast to the normal spirometry/normal DLCO, 10 (22\%) out of 46 active smokers in the normal spirometry/low DLCO group developed airflow limitation consistent with the GOLD criteria for COPD [3] (FEV $1 / \mathrm{FVC}<0.7$ ), nine with GOLD I (FEV1 $\geqslant 80 \%$ prediced post-bronchodilators) and one with GOLD II (FEV1 $\geqslant 50-79 \%$ predicted) $(\mathrm{p}<0.009)$ (figure $2 \mathrm{~d}$ and table 2$)$.

Comparison of the last lung function assessment to the baseline lung function within the normal spirometry/normal DLCO group showed no significant difference in the FEV1 or FVC \% predicted ( $\mathrm{p}>0.3$ for both comparisons) but a significant decrease in the DLCO \% predicted and FEV1/FVC \% observed ( $\mathrm{p}<10^{-4}$ for both comparisons) (figure $3 \mathrm{a}, \mathrm{c}$, e and $\mathrm{g}$ ). We did not assess whether this was or was not

TABLE 2 Progression to chronic obstructive pulmonary disease (COPD) in active smokers with normal spirometry/low diffusing capacity of the lung for carbon monoxide ( $D$ LCo) versus active smokers with normal spirometry/normal DLCo

\begin{tabular}{llc} 
Group & \multicolumn{2}{c}{ At end of evaluation period } \\
\cline { 2 - 3 } & Normal & With COPD \\
\hline $\begin{array}{l}\text { Normal spirometry, normal DLco } \\
\text { Normal spirometry, low DLco } \\
\text { p-value }\end{array}$ & $97(57 / 59)$ & $3(2 / 59)$ \\
& $78(36 / 46)$ & $22(10 / 46)$ \\
\end{tabular}

Data are presented as \% (n/N) unless otherwise stated. 59 active smokers with normal spirometry/normal $D \mathrm{LCO}$ and 46 active smokers with normal spirometry/low D LCO were followed over time with full lung function studies to determine the rate of progression to COPD. \#: individuals with normal spirometry and lung volumes, and normal $D\llcorner C 0$ were followed for mean \pm SD $45 \pm 20$ months; individuals with normal spirometry and lung volumes but low $D$ LCO were followed for $41 \pm 31$ months ( $p>0.4)$. ". : Chi-squared test. 
Normal spirometry, normal

DLCO at baseline
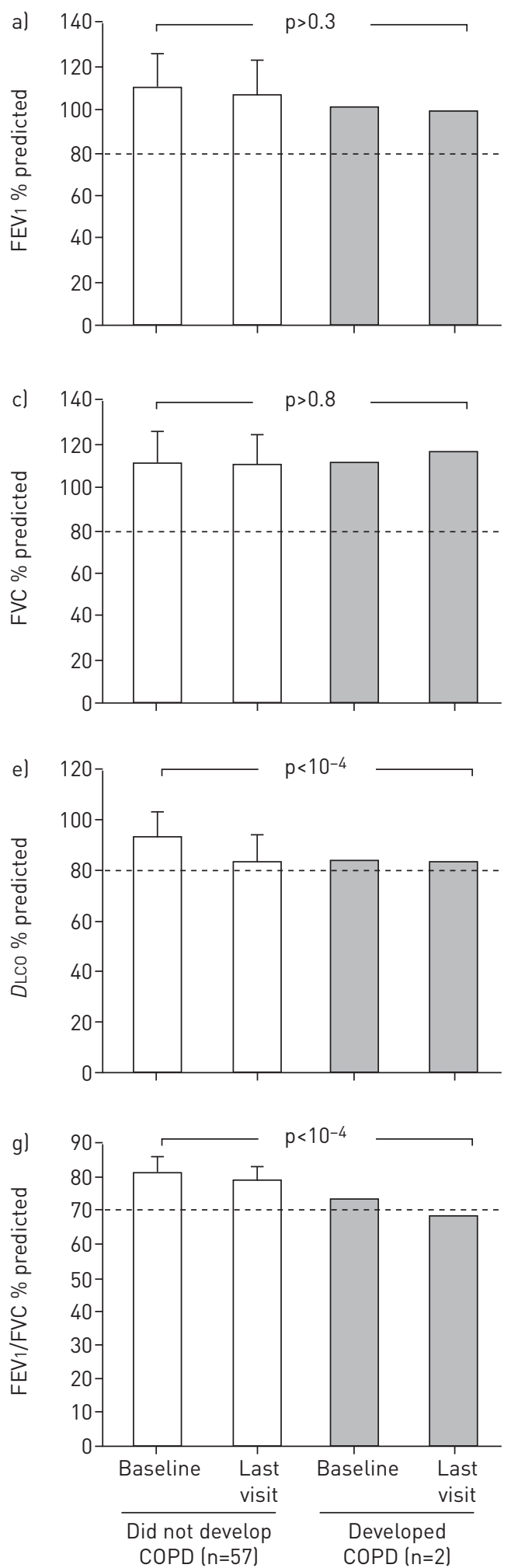

Normal spirometry, low

DLCO at baseline

b)

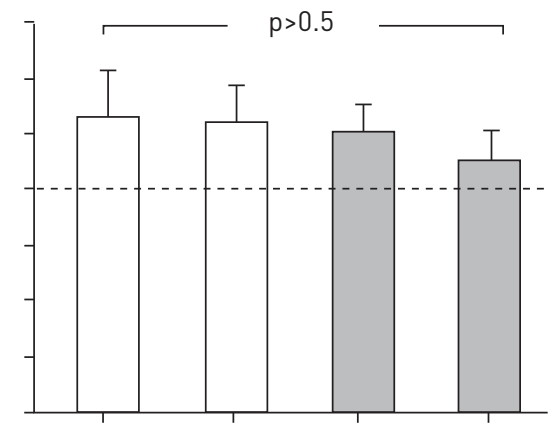

d)

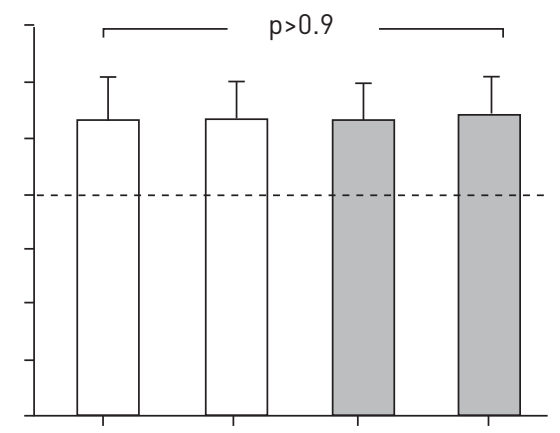

f)

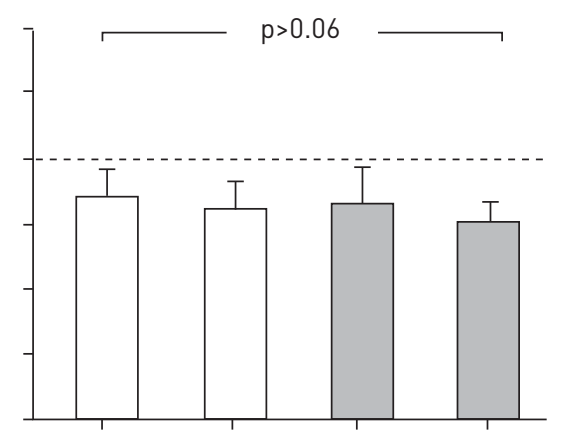

h)

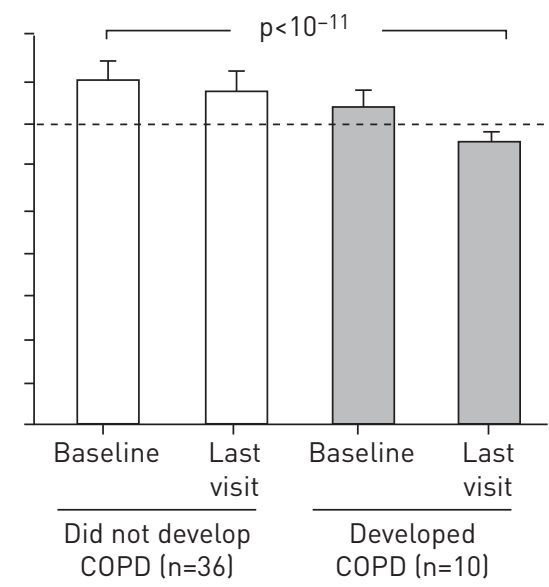

FIGURE 3 Lung function changes from baseline to the last pulmonary function test in the normal spirometry/ normal diffusing capacity of the lung for carbon monoxide ( $D\llcorner\mathrm{LC}$ ) group and normal spirometry/low $D$ Lco group comparing individuals who did not develop chronic obstructive pulmonary disease (COPD) to those who did. $a$ and b) Forced expiratory volume in $1 \mathrm{~s}\left(\mathrm{FEV}_{1}\right)$; $\mathrm{c}$ and d) forced vital capacity (FVC); e and f) DLCo; $g$ and h) FEV $1 / F V C$. Data are presented as mean \pm SD. 
associated with symptoms such as cough, sputum or dyspnoea at the last time-point. Comparison of the last lung function to the baseline lung function within the normal spirometry/low DLCO group showed no change in FEV1, FVC or DLCO \% predicted ( $\mathrm{p}>0.06$ for all comparisons) but a significant reduction in $\mathrm{FEV} 1 / \mathrm{FVC} \%$ observed $\left(\mathrm{p}<10^{-11}\right.$ ) (figure $3 \mathrm{~b}, \mathrm{~d}, \mathrm{f}$ and $\mathrm{h}$ ). Comparison of the rate of change of the $\mathrm{FEV} 1 /$ FVC over time from baseline to last assessment of the normal spirometry/normal DLCO group to the normal spirometry/low DLCO group showed a significantly greater decrease over time for the normal spirometry/low DLCO group (normal spirometry/low DLCO $-0.14 \pm 0.18 \%$ change in FEV1/FVC per month, normal spirometry/normal DLCO $-0.07 \pm 0.11 \%$ change per month; $\mathrm{p}<0.02$ ).

Assessment of the 46 smokers with normal spirometry/low DLCO who were followed over time showed that the distribution of males to females and African-Americans to Europeans or Hispanics was similar in the 10 individuals who developed COPD versus the 36 who did not (supplementary table I). The smoking history, cough and sputum scores, and MMRC scale and serology were also similar in both groups and the age at the last assessment was similar ( $54 \pm 7$ versus $48 \pm 9$ years, respectively; $\mathrm{p}>0.09$ ). Percentage emphysema assessed by HRCT was not significantly different between the groups $(\mathrm{p}>0.05)$. The 10 individuals who developed COPD had lower, but within the normal range, FEV1/FVC $\%$ observed at baseline compared to the 36 individuals who did not developed COPD $(\mathrm{p}<0.003)$. All other lung function parameters were similar between the two groups ( $p>0.05$, all comparisons). On the average, there were no differences in the time of follow-up, number of lung function tests or intervals between lung function tests ( $p>0.1$ for all comparisons). There were no significant differences in any of the parameters or in the prevalence of COPD development between African-Americans, Europeans or Hispanics within and between the low-DLCO smokers who developed COPD and those who did not $(\mathrm{p}>0.09$ for all comparisosns). The assessment of using DLCO levels at baseline as a predictor for development of COPD yielded an area under the curve score of 0.75 ; i.e., DLCO levels can be used to predict COPD development within 41 months with accuracy of $75 \%$.

In addition to using a cut-off of $\mathrm{FEV}_{1} / \mathrm{FVC}<0.7$ to define developing $\mathrm{COPD}$ and $\mathrm{DLCO}<80 \%$ predicted to define low DLCO, a 95\% range of normal DLCO \% predicted and FEV1/FVC [12] was calculated based on the lung function of a 405 healthy nonsmoker dataset (online supplementary material) and used to compare the study population prevalence of developing COPD. Using the normal range for FEV1/FVC and DLCO \% predicted calculated for each sex and ethnicity based on this dataset yielded the same results, with significantly higher prevalence of developing COPD (defined as FEV1/FVC $<95 \%$ normal) in the normal spirometry/low DLCO group versus the normal spirometry/normal DLCO group (low DLCO defined as below the 95\% range).

\section{Discussion}

Cigarette smoking represents the major risk factor for the development of COPD, although only a fraction of smokers develop the disease $[1,2,5,6,16]$. Identification of those smokers at higher risk represents an important step in that the early detection of COPD leads to early therapeutic intervention [1, 2, 17]. Spirometry with bronchodilators is the gold standard tool to screen smokers for COPD [1]. In this study, we focussed on evaluating the addition of the DLCO parameter to identify smokers at risk of the development of COPD. We observed that in a population of 2302 active smokers randomly recruited in the New York metropolitan area responding to advertisements to assess lung health in active cigarette smokers, $17 \%$ had the phenotype of normal spirometry/low DLCO, i.e., the phenotype of low DLCO is quite common among active smokers with normal spirometry. Strikingly, of 105 active smokers randomly chosen for follow-up lung function studies over an average of $<4$ years, $22 \%$ with the normal spirometry/ low DLCO phenotype developed COPD by the GOLD criteria, compared to only $3 \%$ of the normal spirometry/normal DLCO phenotype. These observations suggest that the normal spirometry/low DLCO phenotype is at higher risk for developing COPD than normal spirometry/normal DLCO.

\section{Low DLco in otherwise healthy smokers}

DLCO assesses the potential of the lung for gas exchange [18]. A pathologic correlate of decreased DLCO in smokers is the destruction of the pulmonary capillary bed and a low DLCO in the context of a normal TLC suggests alveolar destruction, i.e. emphysema [8, 18]. A good correlation between low DLCO and emphysema on chest computed tomography has been reported [19, 20]. Consistent with these observations, active smokers with normal spirometry but low DLCO have high circulating levels of endothelial microparticles derived from apoptotic pulmonary capillary endothelium [21]. Decreased DLCO has also been correlated with small airway disease in the presence of severe expiratory airflow limitation and hyperinflation [22]

Our observation that $17 \%$ of active smokers responding to advertisements to assess lung health had a normal spirometry/low DLCO phenotype suggests that, despite a normal spirometry, a significant number of active smokers have a low DLCO, an observation consistent with a number of other studies. Interestingly, 
while the phenotype of smokers with normal spirometry but low DLCO is recognised, there are no data regarding what happens to lung function over time in these individuals.

\section{Risk markers for COPD in smokers}

Identification of markers that trigger early intervention in smokers is important in that even mild COPD is associated with increased mortality [23]. Parameters that help identify the "most vulnerable" smokers, include age, sex, cough, sputum production, dyspnoea, continuation of smoking and pack-years of exposure $[1,2,5,6,14,24-30]$.

In smokers, the prevalence of COPD increases with age [6]. A 25-year follow-up study found that the incidence of COPD in active smokers was $35.5 \%$, with age being a significant predictor for the development of COPD [5]. Advanced age was found to be significantly related to the incidence of COPD in 7- and 10 -year follow-up studies $[28,29]$. In the present study, there was no difference in age between the normal spirometry/normal DLCO and normal spirometry/low DLCO groups or within the normal spirometry/low DLCO group, when comparing the individuals who developed COPD and those who did not.

In addition to age, cough and sputum production have been found by prospective studies to identify individuals with higher risk of developing COPD [26, 28]. A study of Japanese male smokers and nonsmokers demonstrated that productive cough was an independent risk factor for the development of COPD [30]. These data contrast with the studies by FleTCher et al. [27] and VestBo et al. [16], which found that mucus hypersecretion in smokers is a benign condition. In our study, there were no differences in cough and sputum scores between the active smokers with normal spirometry/low DLCO and normal spirometry/ normal DLCO. Furthermore, the individuals followed over time with normal spirometry/low DLCO who developed COPD did not differ in terms of symptoms compared to those who did not develop COPD.

The data pertaining to sex in the development of COPD are conflicting. Studies of smokers, ex-smokers and nonsmokers over 7 and 10 years did not identify sex as a risk factor [28, 29]. However, a study using the GOLD criteria found that despite similar smoking history, men are more susceptible to development of COPD [25] and male smokers have more emphysema than female smokers [24]. In the present study, the development of COPD was sex-independent.

All individuals in our study continued to be active smokers. Continuation of smoking has been found to be an important risk factor for the development of COPD. In the Lung Health Study, smoking cessation significantly slowed the progression to COPD $[1,2,5,17]$.

\section{Implications}

The central observation in this study is that, among active smokers with normal spirometry and normal lung volumes, a decreased DLCO is a risk factor for progression to COPD. These observations need to be verified by larger, randomised trials. Furthermore, the identification of the low- DLCO phenotype is complicated by ethnic variations in "normal" DLCO and significant attention must be paid to quality control. However, with these caveats, the concept that active smokers with normal spirometry/low DLCO are at significantly higher risk for the development of COPD over an average period of $<4$ years than a comparable group of active smokers with normal spirometry/normal DLCO has important implications.

First, the data suggest that DLCO measurement could be an additional tool for early detection of the smoker at risk for COPD, and thus help contribute to early intervention.

Second, while the measurement of DLCO is not presently suitable for routine screening, engineering technology could be developed to make DLCO an early, inexpensive, reproducible measurement, suitable for routine office visits and field use for epidemiological studies.

Third, in the past, DLCO has not been measured in large epidemiological studies such as SPIROMICS and COPDGene [31, 32]. While there are many reasons for this (mostly cost), the observation that a significant percentage of active smokers have a low DLCO and, of these, a significant percentage will develop COPD in an average of $<4$ years has significant implications for the "risk for COPD" parameters assessed in these studies.

Finally, the findings suggest that in smokers, a normal spirometry post-bronchodilator test may give a false sense of "normal", in that a significant subgroup may have a low DLCO and that subgroup is at a significant risk for developing COPD with obstruction.

\section{Acknowledgements}

We thank A. Tilley, S. Hyde and C. Gordon for help with this study, and N. Mohamed (all Department of Genetic Medicine, Weill Cornell Medical College, New York, NY, USA) for help in preparing this manuscript. 


\section{References}

1 Rabe KF, Hurd S, Anzueto A, et al. Global strategy for the diagnosis, management, and prevention of chronic obstructive pulmonary disease: GOLD executive summary. Am J Respir Crit Care Med 2007; 176: 532-555.

2 Mannino DM, Buist AS. Global burden of COPD: risk factors, prevalence, and future trends. Lancet 2007; 370: $765-773$.

3 Global Initiative for Chronic Obstructive Lung Disease. The Global Strategy for the Diagnosis, Management and Prevention of COPD. www.goldcopd.org/uploads/users/files/GOLD_Report_2015_Sept2.pdf Date last accessed: June 28, 2015. Date last updated: September 2, 2015.

4 de Marco R, Accordini S, Cerveri I, et al. An international survey of chronic obstructive pulmonary disease in young adults according to GOLD stages. Thorax 2004; 59: 120-125.

5 Lokke A, Lange P, Scharling H, et al. Developing COPD: a 25 year follow up study of the general population. Thorax 2006; 61: 935-939.

6 Lundback B, Lindberg A, Lindstrom M, et al. Not 15 but 50\% of smokers develop COPD? Report from the Obstructive Lung Disease in Northern Sweden Studies. Respir Med 2003; 97: 115-122.

7 Clark KD, Wardrobe-Wong N, Elliott JJ, et al. Patterns of lung disease in a "normal” smoking population: are emphysema and airflow obstruction found together? Chest 2001; 120: 743-747.

8 Hogg JC. Pathophysiology of airflow limitation in chronic obstructive pulmonary disease. Lancet 2004; 364: 709-721.

9 Klein JS, Gamsu G, Webb WR, et al. High-resolution CT diagnosis of emphysema in symptomatic patients with normal chest radiographs and isolated low diffusing capacity. Radiology 1992; 182: 817-821.

10 Matheson MC, Raven J, Johns DP, et al. Associations between reduced diffusing capacity and airflow obstruction in community-based subjects. Respir Med 2007; 101: 1730-1737.

11 Macintyre N, Crapo RO, Viegi G, et al. Standardisation of the single-breath determination of carbon monoxide uptake in the lung. Eur Respir J 2005; 26: 720-735.

12 Lung function testing: selection of reference values and interpretative strategies. American Thoracic Society. Am Rev Respir Dis 1991; 144: 1202-1218.

13 Miller MR, Hankinson J, Brusasco V, et al. Standardisation of spirometry. Eur Respir J 2005; 26: 319-338.

14 Fletcher CM, Elemes PC, Fairbairn AS, et al. The significance of respiratory symptoms and the diagnosis of chronic bronchitis in a working population. $\mathrm{Br}$ Med J 1959; 2: 257-266.

15 Heijdra YF, Pinto-Plata VM, Kenney LA, et al. Cough and phlegm are important predictors of health status in smokers without COPD. Chest 2002; 121: 1427-1433.

16 Vestbo J, Lange P. Can GOLD stage 0 provide information of prognostic value in chronic obstructive pulmonary disease? Am J Respir Crit Care Med 2002; 166: 329-332.

17 Anthonisen NR, Connett JE, Murray RP. Smoking and lung function of Lung Health Study participants after 11 years. Am J Respir Crit Care Med 2002; 166: 675-679.

18 Crapo RO, Jensen RL, Wanger JS. Single-breath carbon monoxide diffusing capacity. Clin Chest Med 2001; 22: 637-649.

19 Coxson HO, Rogers RM, Whittall KP, et al. A quantification of the lung surface area in emphysema using computed tomography. Am J Respir Crit Care Med 1999; 159: 851-856.

20 Gurney JW, Jones KK, Robbins RA, et al. Regional distribution of emphysema: correlation of high-resolution CT with pulmonary function tests in unselected smokers. Radiology 1992; 183: 457-463.

21 Graham BL, Mink JT, Cotton DJ. Effects of increasing carboxyhemoglobin on the single breath carbon monoxide diffusing capacity. Am J Respir Crit Care Med 2002; 165: 1504-1510.

22 Gelb AF, Zamel N, Hogg JC, et al. Pseudophysiologic emphysema resulting from severe small-airways disease. Am J Respir Crit Care Med 1998; 158: 815-819.

23 Mannino DM, Buist AS, Petty TL, et al. Lung function and mortality in the United States: data from the First National Health and Nutrition Examination Survey follow up study. Thorax 2003; 58: 388-393.

24 Camp PG, Coxson HO, Levy RD, et al. Sex differences in emphysema and airway disease in smokers. Chest 2009; 136: $1480-1488$

25 de Torres JP, Campo A, Casanova C, et al. Gender and chronic obstructive pulmonary disease in high-risk smokers. Respiration 2006; 73: 306-310.

26 de Marco R, Accordini S, Cerveri I, et al. Incidence of chronic obstructive pulmonary disease in a cohort of young adults according to the presence of chronic cough and phlegm. Am J Respir Crit Care Med 2007; 175: 32-39.

27 Fletcher C, Peto R. The natural history of chronic airflow obstruction. Br Med J 1977; 1: 1645-1648.

28 Lindberg A, Jonsson AC, Ronmark E, et al. Ten-year cumulative incidence of COPD and risk factors for incident disease in a symptomatic cohort. Chest 2005; 127: 1544-1552.

29 Lindberg A, Eriksson B, Larsson LG, et al. Seven-year cumulative incidence of COPD in an age-stratified general population sample. Chest 2006; 129: 879-885.

30 Yamane T, Hattori N, Kitahara Y, et al. Productive cough is an independent risk factor for the development of COPD in former smokers. Respirology 2010; 15: 313-318.

31 Couper D, Lavange LM, Han M, et al. Design of the Subpopulations and Intermediate Outcomes in COPD Study (SPIROMICS). Thorax 2014; 69: 491-494.

32 Hersh CP, Washko GR, Estepar RS, et al. Paired inspiratory-expiratory chest CT scans to assess for small airways disease in COPD. Respir Res 2013; 14: 42. 